\title{
The Effectiveness of Local Conservative Therapy after Panretinal Laser Coagulation against the Background of Diabetic Neuropathy
}

\author{
Maria N. Ponomareva ${ }^{1 *}, \mathrm{PhD}, \mathrm{ScD}$; Ivan M. Petrov ${ }^{1}, \mathrm{PhD}, \mathrm{ScD}$; Evgeniya K. Gribanova ${ }^{2}$ \\ ${ }^{1}$ Tyumen State Medical University \\ ${ }^{2}$ Multifunctional clinical diagnostic center \\ Tyumen, the Russian Federation
}

\begin{abstract}
Background: Panretinal photocoagulation (PRP) (also called scatter laser treatment) remains one of the effective methods of treatment, and it can help to prevent blindness and low vision in diabetic retinopathy (DR). The aim of this study was to investigate the efficacy of local prolonged conservative therapy after PRP and the effect of somatic polymorbidity on visual functions in patients with diabetic neuropathy $(\mathrm{DN})$ at the stage of clinical manifestations based on monitoring the clinical, functional and morphometric parameters of the macular region of the retina.

Methods and Results: The study included 78 patients with type 2 diabetes (T2D) who underwent PRP for DR using a VISULAS ${ }^{\circledR}$ 532s solid-state laser (ZEISS). The patients were divided into two groups depending on the presence or absence of DN. Group 1 ( $\mathrm{n}=60,120$ eyes) included patients with DN (stage of clinical manifestations), Group 2 ( $\mathrm{n}=18,36$ eyes) included patients without DN. All patients underwent standard ophthalmological examination: visometry, tonometry, perimetry, biomicroscopy of the anterior segment of the eye and vitreous body, and fundus ophthalmoscopy. Thickness map of the retina was obtained using the RTVue-100 OCT (Optovue, Fremont, CA) EMM5 scan protocol and the Stratus OCT (Carl Zeiss Meditec, USA) radial scan

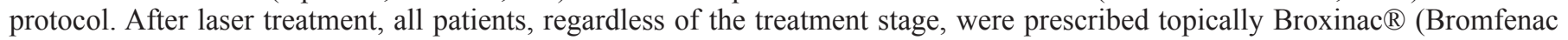
ophthalmic solution $0.09 \%$ ), 1 drop twice a day for a month. In the presence of macular edema, a carbonic anhydrase inhibitor (Dorzolamide $2 \%$ solution) was added to the Broxinac ${ }^{\circledR}$ solution (1 drop twice a day) for up to 1 month after each PRP stage. The dynamics of the parameters of corrected visual acuity and the retinal thickness of the macular region were assessed before PRP and 3 months after the complex treatment.

In patients of Group 1, the dynamics of visual functions against the background of combined treatment (laser and drug) depended on the stage of CKD. Based on the monitoring of the clinical, functional and morphometric parameters of the macular region of the retina after PRP in T2D patients, we found a local, prolonged, 3-month conservative therapy to be effective, using the instillation of Broxinac ${ }^{\circledR}$ supplemented with Dorzolamide $2 \%$ solution in the presence of macular edema. Conversely, there is a negative effect of somatic polymorbidity (stage 3 chronic kidney disease), aspartate aminotransferase $>40 \mathrm{U} / \mathrm{L}$ ) on corrected visual acuity and morphometric parameters of the macular region of the retina during PRP in T2D patients with DN at the stage of clinical manifestations.(International Journal of Biomedicine. 2021;11(3):271-274.)
\end{abstract}

Key Words: diabetes mellitus $\bullet$ diabetic neuropathy $\bullet$ panretinal photocoagulation

For citation: Ponomareva MN, Petrov IM, Gribanova EK. The Effectiveness of Local Conservative Therapy after Panretinal Laser Coagulation against the Background of Diabetic Neuropathy. International Journal of Biomedicine. 2021;11(3):271-274. doi:10.21103/Article11(3)_OA3

\section{Abbreviations}

AH, arterial hypertension; AST, aspartate aminotransferase; BMI, body mass index; CKD, chronic kidney disease; CHD, coronary heart disease; CHF, chronic heart failure; CVA, corrected visual acuity; CVD, cerebrovascular disease; DM, diabetes mellitus; DN, diabetic neuropathy; DR, diabetic retinopathy; GFR, glomerular filtration rate; HDL-C, high-density lipoprotein cholesterol; LDL-C, low-density lipoprotein cholesterol; OCT, optical coherence tomography; OS, oculus sinister; OD, oculus dexter; PRP, panretinal photocoagulation; TG, triglycerides; T2D, type 2 diabetes. 


\section{Introduction}

Diabetes mellitus (DM) is one of the most serious chronic diseases that causes a wide range of complications. ${ }^{(1,2)}$ The persistent hyperglycemia can lead to complications affecting eyes, kidneys, nerves, and heart. ${ }^{(2-4)} \mathrm{DM}$ is a strong predictor of cardiovascular morbidity and mortality and is associated with both micro- and macrovascular complications. A history of concomitant pathology (arterial hypertension, impaired blood lipid spectrum, chronic heart failure) aggravates the formation of microangiopathies. ${ }^{(4,5)}$ According to various authors, in economically developed countries, the frequency of polyneuropathy in diabetes is from $10 \%$ to $90 \%$ of observations ${ }^{(6)}$ and directly depends on the duration of the disease. ${ }^{(7)}$ At the initial diagnosis, $\mathrm{DN}$ is detected in $12 \%$ of patients, and after 20 years - in $50 \%$ of patients. ${ }^{(6)}$ Clinical manifestations of DN do not depend on the type of DM. ${ }^{(7,8)}$ With a duration of diabetes for more than 15 years, detectable retinal damage (retinopathy) in $10 \%$ of cases leads to low vision and in $2 \%$ - blindness. ${ }^{(9)}$

Panretinal photocoagulation (PRP) (also called scatter laser treatment) remains one of the effective methods of treatment, and it can help to prevent blindness and low vision in DR. ${ }^{(5,10,11)}$ Medical management in patients after PRP usually includes instillation of non-steroidal anti-inflammatory drugs, and in the presence of macular edema, the use of carbonic anhydrase inhibitors. ${ }^{(10,12)}$ According to modern scientific literature, the duration of taking these drugs is usually limited to one or two weeks. ${ }^{(5,9)}$ Considering that PRP is carried out in the presence of pronounced phenomena of retinal vascular endotheliosis (pre- and proliferative processes), a local drug support should be long-term, during all stages of laser coagulation, followed by prolongation until there is an effective decrease in the severity of macular edema and an increase in CVA.

The aim of this study was to investigate the efficacy of local prolonged conservative therapy after PRP and the effect of somatic polymorbidity on visual functions in patients with $\mathrm{DN}$ at the stage of clinical manifestations based on monitoring the clinical, functional and morphometric parameters of the macular region of the retina.

\section{Materials and Methods}

The study included 78 patients (50 women and 20 men) with T2D who underwent PRP for DR using a VISULAS ${ }^{\circledR}$ 532s solid-state laser (ZEISS). In this study, the DR classification proposed by Kohner and Porta (1992) was used.

Non-proliferative DR was detected in $8(10.2 \%)$ $[\mathrm{OD}] / 10(12.8 \%)[\mathrm{OS}]$ cases, pre-proliferative DR in 48(61.5\%)[OD]/42(53.8\%)[OS] cases, and proliferative DR in $22(28.2 \%)[\mathrm{OD}] / 26(33.3 \%)[\mathrm{OS}]$ cases, respectively. The exclusion criteria were the presence of the inflammatory, post-traumatic, and dystrophic diseases of the eyeball not associated with DM, as well as hereditary and congenital eye pathologies. PRP was carried out according to the standard method, gradually, in three stages; the interval between the stages of laser treatment was 1 month $(29+5$ days $)$. The median age of the patients was $64[50 ; 77]$ years.
The patients were divided into two groups depending on the presence or absence of DN. Group 1 ( $\mathrm{n}=60,120$ eyes) included patients with DN (stage of clinical manifestations), Group 2 ( $\mathrm{n}=18,36$ eyes) included patients without $\mathrm{DN}$. The groups did not differ in gender composition, age, disease duration, or body mass index.

The study was approved by local ethics committee, and written informed consent was obtained from all participants. All patients underwent comprehensive clinical examination. Laboratory methods included a general blood test, general urine analysis, and the assessment of blood levels of creatinine, TG, HDL-C and LDL-C. BMI was calculated using Quetelet's formula (in $\mathrm{kg} / \mathrm{cm}^{2}$ ). All patients underwent ECG and echocardiography.

All patients were examined by a neurologist, therapist, endocrinologist, cardiologist, and podiatrist. DN was diagnosed by a neurologist based on the assessment of various types of sensitivity (pain, tactile, vibration, cold, and heat) using needles, tuning fork, combined scales of various types of sensitivity, and reflexes. All patients underwent neuromyography using Synapsis, a two-channel electroneuromyographic analyzer. The presence of such concomitant diseases as obesity, AH, dyslipidemia and CHF was taken into account. CKD was diagnosed with determination of the blood creatinine level and further calculation of the GFR using the Cockcroft\&Gault formula.

All patients underwent standard ophthalmological examination: visometry, tonometry using non-contact pneumotonometer (Reichert Technologies), perimetry using PNR-2-01, biomicroscopy of the anterior segment of the eye and vitreous body on an SL-140 slit lamp (Carl Zeiss Meditec AG, Germany), and fundus ophthalmoscopy using a noncontact Ocular MaxField High Mag 78D Lens.

Thickness map of the retina was obtained using the RTVue-100 OCT (Optovue, Fremont, CA) EMM5 scan protocol and the Stratus OCT (Carl Zeiss Meditec, USA) radial scan protocol.

After laser treatment, all patients, regardless of the treatment stage, were prescribed topically Broxinac ${ }^{\circledR}$ ( Bromfenac ophthalmic solution $0.09 \%$ ), 1 drop twice a day for a month. In the presence of macular edema, a carbonic anhydrase inhibitor (Dorzolamide 2\% solution) was added to the Broxinac ${ }^{\circledR}$ solution (1 drop twice a day) for up to 1 month after each PRP stage. The dynamics of CVA parameters and the retinal thickness of the macular region were assessed before PRP and 3 months after the complex treatment.

Statistical analysis was performed using IBM SPSS Statistics for Windows, Version 23.0. Armonk, NY: IBM Corp. The normality of distribution of continuous variables was tested by one-sample Kolmogorov-Smirnov test. Continuous variables with normal distribution were presented as mean (standard error of the mean [SEM]); non-normal variables were reported as median (interquartile range (IQR; 25th to 75th percentiles). Student's unpaired and paired $\mathrm{t}$-tests were used to compare two groups for data with normal distribution. Mann-Whitney $U$ test was used to compare means of 2 groups of variables not normally distributed. The Wilcoxon criterion was used to compare the differences 
between the paired samples. The frequencies of categorical variables were compared using Pearson's chi-squared test or Fisher's exact test, when appropriate. A value of $P<0.05$ was considered significant.

\section{Results}

Analysis of the presence of concomitant diseases showed a significant predominance of $\operatorname{CHD}(P=0.037)$ in patients of Group 1 (Table 1). Before treatment, the CVA values between the groups did not differ. After the combined treatment, there was a tendency to an increase in CVA in both groups. Before PRP, there was a weak correlation with the patient age $(\mathrm{r}=0.278, P<0.037)$ in Group 1 and a weak one after PRP $(\mathrm{r}=0.328, P<0.011)$ in Group 2. In patients of Group 1 , the dynamics of visual functions against the background of combined treatment (laser and drug) depended on the stage of CKD (Table 2). The results of our study revealed a weak correlation between CVA and GFR before and after PRP ( $\mathrm{r}=0.260, P<0.04$ and $\mathrm{r}=0.364, P<0.04$, respectively) in patients of Group 1 and a moderate correlation $(\mathrm{r}=0.412$, $P<0.001 / \mathrm{r}=0.492, P<0.001$, respectively) in patients of Group 2. In addition, there was a weak correlation between CVA and AST in both groups $(\mathrm{r}=0.263, P<0.042)$ before PRP.

Table 1.

Concomitant diseases in groups

\begin{tabular}{|l|c|c|c|}
\hline \multicolumn{1}{|c|}{ Disease } & $\begin{array}{c}\text { Group 1 } \\
(\mathrm{n}=60)\end{array}$ & $\begin{array}{c}\text { Group 2 } \\
(\mathrm{n}=18)\end{array}$ & $P$-value \\
\hline $\mathrm{CHD}, \mathrm{n}(\%)$ & $30(50)$ & $4(22.2)$ & 0.037 \\
\hline $\mathrm{CVD}, \mathrm{n}(\%)$ & $10(16.7)$ & $3(16.7)$ & $>0.05$ \\
\hline Obesity, n (\%) & $36(60.0)$ & $10(55.6)$ & $>0.05$ \\
\hline $\mathrm{AH}, \mathrm{n}(\%)$ & $52(86.7)$ & $17(94.4)$ & $>0.05$ \\
\hline $\mathrm{CHF}, \mathrm{n}(\%)$ & $41(68.3)$ & $11(61.1)$ & $>0.05$ \\
\hline
\end{tabular}

Table 2.

The level of CVA depending on the stage of CKD

\begin{tabular}{|l|c|c|c|c|c|c|}
\hline \multirow{2}{*}{$\begin{array}{c}\text { GFR, } \\
\mathrm{ml} / \mathrm{min} / 1.73 \mathrm{~m}^{2}\end{array}$} & \multicolumn{4}{|c|}{ Group 1 } & \multicolumn{3}{c|}{$\begin{array}{c}\text { Group 2 } \\
\text { Pefore } \\
\text { PRP }\end{array}$} & $\begin{array}{c}\text { After } \\
\text { PRP }\end{array}$ & $\mathrm{n}$ & $\begin{array}{c}\text { Before } \\
\text { PRP }\end{array}$ & After PRP \\
\hline $\begin{array}{l}\text { GFR }>90 \\
\text { Stage 1 }\end{array}$ & 46 & $0.51 \pm 0.48$ & $0.73 \pm 0.27 *$ & 20 & $0.57 \pm 0.33$ & $0.66 \pm 0.34 *$ \\
\hline $\begin{array}{l}\text { GFR 60-89 } \\
\text { Stage 2 }\end{array}$ & 40 & $0.51 \pm 0.39$ & $0.68 \pm 0.21$ & 14 & $0.59 \pm 0.25$ & $0.67 \pm 0.28 *$ \\
\hline $\begin{array}{l}\text { GFR 30-59 } \\
\text { Stage 3A/3B }\end{array}$ & 34 & $0.54 \pm 0.17$ & $0.54 \pm 0.15$ & 2 & $0.57 \pm 0.02$ & $0.63 \pm 0.01$ \\
\hline$*$ * $P<0.05$
\end{tabular}

Of greatest interest are the changes in the morphometric parameters of the macular region of the retina after PRP (Table 3). In general, patients in Group 1 showed a tendency towards a decrease in the retinal thickness in all segments. There was a complete regression of macular edema in $15.0 \%$ of cases and a tendency to decrease it in $28.3 \%$ of cases, more pronounced in the central and temporal segments. Patients of Group 2 showed a significant (relative to normal values, $P<0.05$ )) decrease in the retinal thickness in the central, superior and inferior segments. In addition, we observed a complete regression of macular edema in 3 patients of this group. The absence of any dynamics in macular edema was found in $10.0 \%$ of patients in Group 1 and deterioration of the morphometric parameters of the macular region of the retina in $20.0 \%$ of patients with CKD Stage 3A/3B and increased AST level (>40 U/1)..

Domestic and foreign scientists have demonstrated the effect of comorbidity on the development of DN and DR. ${ }^{(4,7)}$ Our study showed that diabetic macroangiopathies, concomitant diseases (arterial hypertension, chronic heart failure), occur with almost the same frequency in both groups, without significantly affecting the development of DN at the stage of clinical manifestations. CHD is the trigger causes for the development of DN at the stage of clinical manifestations.

Table 3.

The retinal thickness $(\mu m)$ before and after PRP

\begin{tabular}{|c|c|c|c|c|}
\hline \multirow{2}{*}{ Segment } & \multicolumn{2}{|c|}{ Group 1} & \multicolumn{2}{|c|}{ Group 2} \\
\hline & Before PRP & After PRP & Before PRP & After PRP \\
\hline $\begin{array}{l}\text { Central } \\
\text { OD } \\
\text { OS }\end{array}$ & $\begin{array}{l}230.95 \pm 69.12 \\
230.10 \pm 69.54\end{array}$ & $\begin{array}{l}220.81 \pm 64.25 \\
220.20 \pm 81.14\end{array}$ & $\begin{array}{c}213.0 \pm 84.0 \\
210.83 \pm 43.28\end{array}$ & $\begin{array}{l}207.34+11.21 * \\
203.89 \pm 12.11^{*}\end{array}$ \\
\hline $\begin{array}{l}\text { Nasal } \\
\text { OD } \\
\text { OS }\end{array}$ & $\begin{array}{l}266.83 \pm 29.89 \\
299.90 \pm 16.94\end{array}$ & $\begin{array}{l}267.01 \pm 28.16 \\
262.46 \pm 33.11\end{array}$ & $\begin{array}{c}265.33 \pm 30.0 \\
265.77 \pm 25.35\end{array}$ & $\begin{array}{c}264.0 \pm 29.16 \\
258.27 \pm 25.62\end{array}$ \\
\hline $\begin{array}{l}\text { Superior } \\
\text { OD } \\
\text { OS }\end{array}$ & $\begin{array}{l}295.91 \pm 157.01 \\
294.51 \pm 154.12\end{array}$ & $\begin{array}{l}286.76 \pm 145.23 \\
285.06 \pm 105.98\end{array}$ & $\begin{array}{l}276.05 \pm 86.51 \\
277.33 \pm 81.25\end{array}$ & $\begin{array}{l}260.66 \pm 29.23 * \\
266.83 \pm 23.05^{*}\end{array}$ \\
\hline $\begin{array}{l}\text { Temporal } \\
\text { OD } \\
\text { OS }\end{array}$ & $\begin{array}{l}296.79 \pm 117.21 \\
298.65 \pm 125.45\end{array}$ & $\begin{array}{l}288.06+93.17 \\
286.16 \pm 99.23\end{array}$ & $\begin{array}{l}271.22+47.58 \\
267.83 \pm 57.45\end{array}$ & $\begin{array}{l}271.27+44.23 \\
263.83 \pm 44.52\end{array}$ \\
\hline $\begin{array}{l}\text { Inferior } \\
\text { OD } \\
\text { OS }\end{array}$ & $\begin{array}{l}286.22 \pm 131.14 \\
283.95 \pm 111.54\end{array}$ & $\begin{array}{c}279.65 \pm 111.65 \\
276.43 \pm 87.56\end{array}$ & $\begin{array}{c}274.72+102.56 \\
268.95 \pm 26.85\end{array}$ & $\begin{array}{c}265.95+32.56 \\
263.72 \pm 27.56^{*}\end{array}$ \\
\hline
\end{tabular}

*. $P<0.05$ 
We revealed that the CVA level after PRP against the background of long-term local treatment in T2D patients with $\mathrm{DN}$ at the stage of clinical manifestations depends on age and the functional state of the liver and kidneys. The combined treatment of DR is not effective in the presence of macular edema, $\mathrm{CKD} 3 \mathrm{~A} / 3 \mathrm{~B}$, or changes in the reference parameters of AST and GFR. These criteria are biological markers of a poor prognosis for CVA after PRP and prolonged local conservative treatment. The results obtained require additional research and development of an algorithm for preliminary correction of somatic status in order to increase the effectiveness of PRP in preventing blindness and low vision in this category of patients. Prolonged administration of Broxinac $₫$ in Group 2 patients without DN and macular edema is effective in relieving possible complications after PRP. In cases where macular edema is detected in patients without $\mathrm{DN}$, it is also advisable to prescribe Dorzolamide 2\%.

In conclusion, based on the monitoring of the clinical, functional and morphometric parameters of the macular region of the retina after PRP in T2D patients, we found a local, prolonged, 3-month conservative therapy to be effective, using the instillation of Broxinac $₫$ supplemented with Dorzolamide $2 \%$ solution in the presence of macular edema. Conversely, there is a negative effect of somatic polymorbidity (CKD Stage 3, AST $>40 \mathrm{U} / \mathrm{L}$ ) on CVA and morphometric parameters of the macular region of the retina during PRP in T2D patients with $\mathrm{DN}$ at the stage of clinical manifestations.

\section{Competing Interests}

The authors declare that they have no competing interests.

\section{References}

1. Standards of specialized diabetes care. Edited by Dedov II, Shestakova MV, Mayorov AYu. The 9th edition. Diabetes Mellitus. 2019;22(1S1). doi: 10.14341/DM20191S1. [In Russian].

*Corresponding author: Prof. Maria N. Ponomareva, PhD, ScD. Tyumen State Medical University. Tyumen, the Russian Federation.E-mail: e-mail: mariyponomareva@yandex.ru
2. Introduction: Standards of Medical Care in Diabetes-2018. Diabetes Care. 2018 Jan;41(Suppl 1):S1-S2. doi: 10.2337/ dc18-Sint01.

3. Diabetes mellitus: diagnosis, treatment, prevention. Ed. Dedova II, Shestakova MV. Moscow: Medical Information Agency, 2011. [In Russian].

4. Trukhan DI, Lebedev OI. Izmenenie organa zreniia pri somaticheskikh zabolevaniiakh [Change in the organ of vision in somatic diseases]. Ter Arkh. 2015;87(8):132-136. doi: 10.17116/terarkh2015878132-136. [Article in Russian].

5. Kazaykin VN. Diabetic retinopathy: clinical presentation, diagnosis, and treatment. Moscow: Medinform, 2016. [In Russian].

6. Khramilip VN, Demidova IU, Ignatova OY. [Prevalence of diabetic polyneuropathy in newly diagnosed type 2 diabetes mellitus]. Vrach (The Doctor). 2009;5:40-43. [Article in Russian].

7. Al-Zamil MH, Kochetova OV. [Diabetes mellitus and diabetic neuropathy: a historical aspect]. Klinicheskaia Nevrologiia. 2009;3:45-47. [Article in Russian].

8. Meerwaldt R, Links TP, Graaff R, Hoogenberg K, Lefrandt JD, Baynes JW, Gans RO, Smit AJ. Increased accumulation of skin advanced glycation end-products precedes and correlates with clinical manifestation of diabetic neuropathy. Diabetologia. 2005 Aug;48(8):1637-44. doi: 10.1007/s00125005-1828-x.

9. Chew EY, Davis MD, Danis RP, Lovato JF, Perdue LH, Greven C, et al.; Action to Control Cardiovascular Risk in Diabetes Eye Study Research Group. The effects of medical management on the progression of diabetic retinopathy in persons with type 2 diabetes: the Action to Control Cardiovascular Risk in Diabetes (ACCORD) Eye Study. Ophthalmology. 2014 Dec;121(12):2443-51. doi: 10.1016/j. ophtha.2014.07.019.

10. Bobykin EV. [Current approaches to the treatment of diabetic macular edema. A literature review]. Fyodorov Journal of Ophthalmic Surgery. 2019;(1):67-76. doi: 10.25276/02354160-2019-1-67-76. [Article in Russian].

11. Mozherencov VP, Prokofyeva GL, Usova LA. [Ocular manifestations of diabetes mellitus]. Russian Journal of Clinical Ophthalmology. 2002;1:31. [Article in Russian].

12. Astakhov YuS, Shadrichev FE, Krasavina MI, Grigorieva NN. [Modern approaches to the treatment of diabetic macular edema]. Ophthalmology Journal. 2009;2(4): 59-69. [Article in Russian]. 